\title{
Digital Smart Pen: A Portable Media with Endless Benefits
}

\author{
Samuel Babafemi Olabisi \\ Department of Computer Science \\ Tai Solarin College of Education \\ Omu - ljebu, Nigeria
}

\author{
Aderibigbe Adesoji David \\ Department of General Studies \\ Tai Solarin College of Education \\ Omu - ljebu, Nigeria
}

\begin{abstract}
The purpose of education and learning is often defeated when gadgets developed to assist in the process of learning is not user friendly or ends up becoming a distraction in the classroom due to its size.

This work intends to describe a gadget known as smart pen which is a new and evolving technology that does not create distraction due to its portability, that is user friendly and above all its affordable nature.

Just like any other pen, it is a writing device developed by the livescribe company. It is a digital and intelligent pen that is fast gaining recognition in synchronizing written notes with audio contents.
\end{abstract}

\section{General Terms}

Smart Pen, Livescribe

\section{Keywords}

Pictorial Representation of a smart pen, Pencasting, Intelligent Pen, Evolving Technology.

\section{INTRODUCTION}

Smart pen is similar to an ordinary pen but it is a digital device with high technology writing tool that records spoken words and synchronizes them with notes written by a user on a special paper. The special paper used is known as dot paper notebook. The smart pen can be connected to a computer to upload the recorded data for saving and sharing [11]. By tapping the pen's tip, students can replay back everything a teacher has said in the classroom [3].

The most popular type of smart pen is the echo and pulse from livescribe. The pulse smart pen was introduced in March 2008 with 2GB to 4GB memory, built in speaker, OLED display, Infrared camera and USB connector while the echo smart pen was introduced in mid-2010 with advanced features to replace the audio and data ports of the pulse smart pen with a standard headphone and micro-USB port [1].

It is on record that five hundred thousand of livescribe pulse and echo smart pens was sold at the end of year 2010.It comes in color black with a rounded bottom that is thick but the top being flat for the OLED display to be installed within it [9].

There are so many applications available on the smart pen which consists of language applications to make translation easier, dictionary to assist in studying and learning, games to create entertainment etc. Indeed with these features of a smart pen, one can say authoritatively that it is a great and intelligent pen worth having as a companion [6].

\section{SMART PEN}

\subsection{Brief History}

Jim Margraff [7] was the inventor of Leapfrog FLY pen top computer and Leap PAD learning system, a trademark under the Leapfrog Company that was popular for its interactive children books which showed the capacity to link audio to paper at the touch of a pen.

In 2005, Margraff left his successful business (Leapfrog Company) and diversified into a new project which will later metamorphose into the livescribe company in January 2007. His vision was to invent an electronic pen that would record written and oral information at any time [7].

Today that pen is referred to as a smart pen; a trademark for which livescribe is noted for worldwide. Livescribe's headquarter is based in Oakland, California in United States of America and as at year 2011, it has close to 100 employees [11].

\subsection{Pictorial Representation of a Smart Pen}

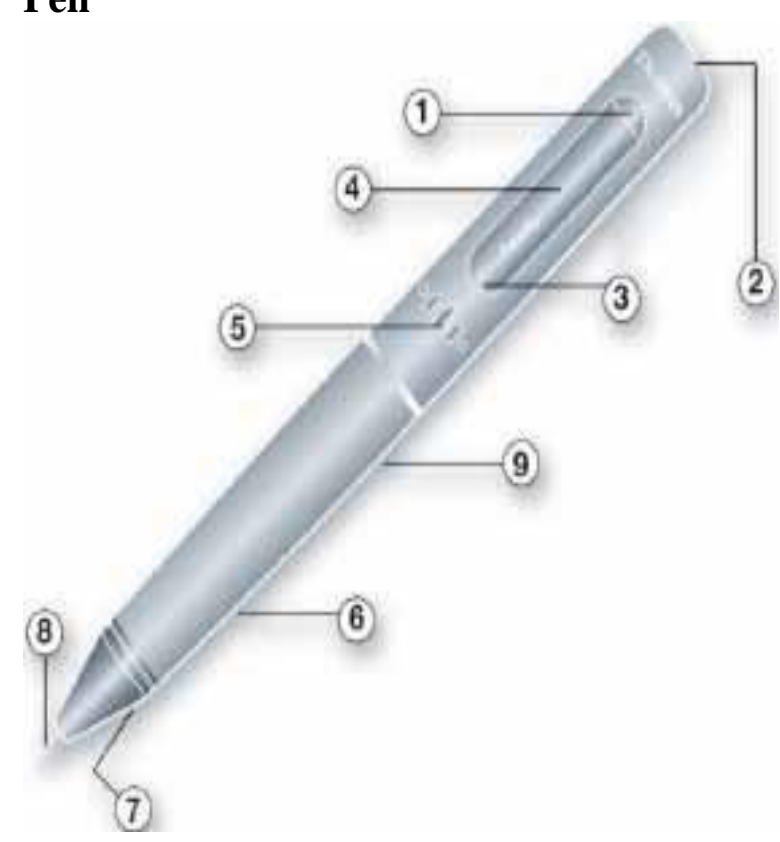

Figure A 
1. Power button: The power button turns on the smart pen. It also allows the user to start recording audio files if it is held down for about two seconds. [13]

2. Headset jack: The headset contains an ear bud with an embedded microphone that enables the smart pen record quality sound in a noisy environment.

3. Built-in microphone: Each side of the OLED display has two microphones built in it; this allows the smart pen record sharp and clear sounds.

4. OLED display: It is referred to as an organic light emitting diode. This is the interface between the user and smart pen. It allows easy navigation of various applications on the device.

5. Built in speaker: It provides feedback in form of audio for the user during navigation of various applications on the smart pen, also the recorded sounds can be replayed through this medium. [13]

6. USB Connector: When connected to the computer, the USB connector transfers written notes and recorded audio to a PC. It can also serve as a platform to recharge the smart pen.

7. Infrared Camera: Everything you write, tap or draw on the dot paper notebook, the infrared camera keeps track of it. It operates at a very high speed.

8. Ink Cartridge: The ink cartridge of a smart pen is removable and can be replaced when the user realizes that the quality of written notes is becoming vague.

9. Rechargeable lithium battery: The lithium battery is rechargeable but non removable. The life span of the battery determines the continued usage of the smart pen. [13]

\section{EDUCATIONAL BENEFITS OF SMART PEN}

Though smart pen is a new technology evolving but its wide range of uses has made it generally acceptable to various professionals i.e. teachers, lawyers, doctors, secretaries, administrators, journalists, investigation officers etc. Its immense contribution to education cannot be under estimated thus it has become the center of attraction towards producing effective learning process through fast rate of assimilation and high quality of note taking which can be replayed at a convenient time. Below is a list of smart pen uses:

- Pronunciation and oral class: Teachers can record models meant for pronunciation for students to listen to, also pupils can record theirs, then compare it to the teacher's model to see if there is correlation or not. This process helps students to achieve greater oral skills within a shorter period of time [4].

- Pencasting: It is a technique that captures everything you write or draw together with audio contents. Home work can be done by students, then pencast it and the links can be communicated to the teachers [5].

- Calculator: In order to aid arithmetic operations, every smart pen comes with a functional scientific calculator [4]

- For students without access to computer: Students without PC do have equal and ample opportunity to replay and listen to the teacher's explanation over and over again.
- Basic piano skills: It provides the platform to learn keys i.e. c, d, e, f, g, a, b etc. both sharps and flats necessary to play piano cords. [4]

- Affordable and portable: Unlike other gadgets like notebooks, Personal Digital Assistants (PDA) [8] etc. The smart pen is of low cost, handy, portable and simple to use.

- Absent students are not at disadvantage: A detailed explanation of the teacher's lecture can be pencast to students who are absent from school. It provides them chance to listen to the lecture exactly original as it was taught by the teacher in the classroom [4].

- Sharing files: Smart pen comes with a USB port which allows it to be connected to a computer in order to transfer files via blogs, social networks, Emails etc. [2]

- $\quad$ Aids student with hearing disabilities: Students with hearing impediments can keep up with the tempo of lecture by recording the teacher's explanation and using symbols to represent cogent facts on the dot notebook e.g. a leaf to represent photosynthesis. It helps in building their selfconfidence and independence.

- Slow down speed up audio: Teachers lectures can be rewinded or fast forwarded in order to aid comphrension depending on the listening skills of the student [4].

- Substitute lesson plan: Smart pen allows teachers stay in touch with pupils. The teacher can record verbally, a detailed lesson plan and description of classroom procedures, and then leave his substitute to tap and play for students in case of his absence.

- Mapping: It is a graphical representation. The similarities and relevance of major and sub points that support a topic can be illustrated by a map in form of diagrams. Maps help to improve memory retention by classifying materials in a highly visualized manner [5].

- Dictionary: The dictionary comes with a thesaurus and can be downloaded onto the smart pen from the livescribe site, to help students define words to assist in studying and productivity [6].

- Personalization by the user: The microprocessor of the smartpen uses JAVA technology which allows users to download additional software other than the pre-installed ones, thus giving the user wide variety of applications [11].

- Translator: It performs translation by simply selecting the language and then directing the pen over the desired word that the user wants to translate in order to hear it [12]. 


\section{SMART PEN OF THE FUTURE}

Presently the ink cartridge of a smart pen comes in black color, but in the future, it is proposed that smart pen should have multi-colored cartridge which will help the user to change color of notes as he or she wishes. Also it will make note taking more fascinating. The function of the "Jump" button of the smart pen is sometimes confusing and ambiguous particularly for a novice (new user); hence the urge to replace it with a "Play" button which will enhance the replay of audio files more quickly and efficiently. Lastly a wireless smart pen may spring up, this will help the user to transfer files without attaching it to the computer via nonphysical medium. [10]

\section{CONCLUSION}

Students do come from different background, upbringing and individual differences thus it affects their listening skills, perception of new ideas and rate of assimilation in the classroom. The smart pen is like a balance creating a scenario where students need not be worried about the tempo of a lecture by a teacher. The importance and usefulness of smart pen is unquantifiable and not limited to the above discussed benefits in this work. Though it is an evolving technology but its impact in aiding teaching and learning cannot be ignored rather it is fast becoming a gadget that is indispensable.

\section{ACKNOWLEDGEMENTS}

Our sincere appreciation goes to the authors whose work were consulted and livescribe website for providing useful information about their product.

\section{REFERENCES}

[1] Associated, P. 2011. Review: Livescribe Smart Pen is Ipad's opposite. Daily Herald Online, Retrieved from http://www.dailyherald.com/.
[2] Bonnigton, Christian. 23 ${ }^{\text {rd }}$ May, 2011. "Livescribe connect shares smart pen notes to Facebook, E-mail". Retrieved August 19,2012

[3] Computing Assistive Technology; what is a Smart Pen by Andrew Leibs, Retrieved from http://www.about.com/. on $8^{\text {th }}$ May, 2013

[4] Educational uses for the Livescribe pulse Smart Pen, Retrieved online from http://www.livescribe.com/support on $1^{\text {st }}$ May, 2013

[5] Janet Sankar, Education Idea Book V3 Copyright 2011 Livescibe Inc. Retrieved online from http://www.livescribe.com/support.

[6] Livescribe Echo Digital Smart Pen, 2011. Retrieved from http:// www.ezinarticles.com/.

[7] Margraff, J. (19 $9^{\text {th }}$ December, 2010). Interview by M. Chan [Personal Interview], Livescribe's smart pen synchronizes speech with writing. Retrieved from http://articles. Sfgate. Com/

[8] Schaak, A. V (2009) Livescibe in K-12 Education; Research support: A review of scientific evidence demonstrating the effectiveness of smart Pen technology for improving teaching and learning.

[9] Thompson, C (16 ${ }^{\text {th }}$ September, 2010). The pen that never forgets. The New York Times pp. A1, Retrieved from http://www.nytimes.com/.

[10] Vanessa, Berenguer. \& Valter, Candelaria; University of Madeira "Smart Pen", a2035305@ max.uma.pt

[11] http://www.livescribe.com/en-us/; Livescribe Inc. 2011.

[12] http://www.livescibeecho.wordpress.com/; Livescribe Echo Digital Smart pen - The ultimate in personal recordings.

[13] http://www.livescribe.com/support. 